\title{
Specific IgG4 Production during House Dust Mite Immunotherapy among Age, Gender and Allergic Disease Populations
}

\author{
Xuxin Lai ${ }^{a}$ Jing $\mathrm{Li}^{\mathrm{b}}$ Xiaoxiong Xiao ${ }^{c}$ Enmei Liu ${ }^{\mathrm{d}}$ Chunqing Zhang ${ }^{\mathrm{b}}$ \\ Hongyu Wang $^{b}$ Birgitte Gjesing ${ }^{a}$ Nanshan Zhong ${ }^{b}$ Michael D. Spangfort ${ }^{a}$

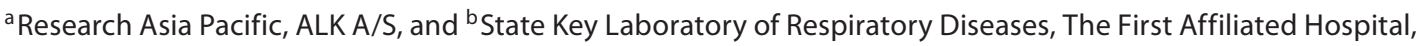 \\ Guangzhou Medical College, Guangzhou, ' Zhongshan Bo Ai Hospital, Zhongshan, and dThe Children's Hospital of \\ Chongqing University of Medical Sciences, Chongqing, China
}

\section{Key Words}

Allergen-specific immunotherapy · House dust mite •

Allergen-specific immunoglobulin $\mathrm{G} 4$

\begin{abstract}
Background: Specific lgG4 induced by allergen-specific immunotherapy (SIT) is an immunological marker related to the appearance of clinical tolerance. But specific lgG4 levels in different age, gender and allergic disease populations have not been fully investigated. Methods: This study involved 226 children and 109 adults with allergic rhinitis and/or asthma receiving a 156-week course of Dermatophagoides pteronyssinus (Der p) subcutaneous SIT. Symptom and medication scores, forced expiratory volume after $1 \mathrm{~s}\left(\mathrm{FEV}_{1}\right)$ and Der $\mathrm{p}$ specific lgG4 levels at weeks 0, 5, 10, 25, 52, 104 and 156 were analyzed. Results: Rhinitis symptom and medication scores and $\mathrm{FEV}_{1} \%$ predicted in children showed significantly greater improvement than in adults at week 104 and 156 ( $p<$ 0.05). Levels of Der $p$-specific lgG4 showed a significant increase after 10 weeks of subcutaneous SIT $(p<0.0001)$ and continued to increase during the 156-week SIT period. Before SIT, the initial Der p-specific lgG4 level was higher in children than adults $(p=0.0004)$. The increase ratio of Der p-specific lgG4 was higher in children than adults at 52
\end{abstract}

weeks $(p<0.001)$ and 104 weeks $(p=0.0156)$ of SIT, and was higher in rhinitis compared to asthma patients at 156 weeks of SIT ( $p=0.0244)$. There was no difference between males and females at any time points. Conclusion: Children are more responsive to SIT, demonstrating clinical and $\mathrm{FEV}_{1}$ improvement and producing higher levels of allergen-specific IgG4 during a shorter SIT period compared to adults. Rhinitis patients show a higher increase in specific lgG4 compared to patients with asthma symptoms. The increase of Der $\mathrm{p}$ specific IgG4 reflects a specific response of the immune system towards the SIT vaccine being administrated.

Copyright @ 2012 S. Karger AG, Basel

\section{Introduction}

Allergic diseases have increased significantly due to the growth of urbanization and changes to environments. Allergen-specific immunotherapy (SIT) using standardized vaccines at high doses is an effective treatment for patients with allergic rhinitis, asthma and stinging insect allergy [1]. SIT is the only treatment at present that can treat the underlying cause and modify the development of disease. In a double-blind, placebo-controlled study of house dust mite immunotherapy in Chinese asthmatic patients, we

\section{KARGER}

Fax +4161306 1234

E-Mail karger@karger.ch

www.karger.com
(C) 2012 S. Karger AG, Basel

$1018-2438 / 13 / 1601-0037 \$ 38.00 / 0$

Accessible online at:

www.karger.com/iaa
Correspondence to: Dr. Jing Li

State Key Laboratory of Respiratory Diseases, The First Affiliated Hospital

Guangzhou Medical College, 151 Yan Jiang Road

Guangzhou 510120 (China)

Tel. +862034296151, E-Mail lijing@gird.cn 
have shown that 1 year of Dermatophagoides pteronyssinus (Der p) immunotherapy significantly reduced symptoms and medication use in asthmatic subjects, and this was associated with a greater subjective improvement in symptom and medication scores of asthma [2].

The mechanisms for successful SIT are still not fully elucidated but several immunological effects, such as the appearance of SIT-induced regulatory T cells, induction of IgG-blocking antibodies and induction of immunotolerance have been reported [3-5]. Successful SIT is always accompanied by a significant increase of specific IgG4 levels. IgG4 is a unique antibody with a Fab-arm exchange ability to generate monovalent and non-crosslinking antibodies [6]. Although there is no clear correlation between serum concentrations of specific IgG4 and clinical efficacy [4], SIT-induced specific IgG4 has been shown to act as a blocking antibody by competing with specific IgE in the binding of allergens affecting mast cells and basophil activity, and interfering with IgE-facilitated antigen presentation, thereby preventing the allergen-dependent activation of $\mathrm{T}$ cells [7-8]. The blocking ability of specific IgG4 is still in effect after completing the course of SIT although the levels of IgG4 have decreased [9]. Specific IgG4 is thus an important immunological marker for SIT when objectively assessing the clinical performance of the treatment.

Der $\mathrm{p}$ and Dermatophagoides farinae (Der f) are the dominating indoor allergen sources in China, having a skin prick test prevalence of about $60 \%$ among allergic patients [10]. The majority of Chinese house dust miteallergic patients are sensitized against both Der $p$ and Der f with nearly $100 \%$ prevalence of IgE sensitization against the Der $\mathrm{p}$ and Der $\mathrm{f}$ major allergens of group 1 and group 2 [11-12]. Despite this apparent dual sensitization, SIT using Der p-standardized vaccine is clinically effective and induces specific IgG4 responses against both mites [13] due to the large degree of sequence and structural homology between allergens from the respective mite species [14].

In this study, 335 patients with allergic rhinitis and/or asthma, who were sensitized against house dust mite, received subcutaneous SIT using a standardized Der $\mathrm{p}$ allergen vaccine. The specific IgG4 levels were measured in 872 serum samples collected at various SIT time points. Through analyzing a large number of sera from patients undergoing standardized Der p SIT, we aimed at finding distribution patterns of Der p-specific IgG4 in populations of different age (children vs. adults), gender (male vs. female) and diseases (allergic rhinitis vs. asthma), along with clinical evaluations.

\section{Materials and Methods}

\section{Subjects}

A total of 335 subjects, 226 children (age $\leq 14$ years) and 109 adults (age 15-64 years), were included in this study: 225 came from the respiratory department of Guangzhou Institute of Respiratory Diseases (116 children and 109 adults), 77 from the pediatric department of Zhongshan Bo Ai Hospital and 33 from Chongqing Children's Hospital. All patients fulfilled the GINA guidelines for mild-to-moderate asthma and ARIA guidelines for allergic rhinitis. All patients were skin prick test positive to Der $\mathrm{p}$ and had specific serum IgE against Der $\mathrm{p}$. The 225 patients from Guangzhou had their forced expiratory volume after $1 \mathrm{~s}\left(\mathrm{FEV}_{1}\right)$ measured, which was required to be $\geq 70 \%$ predicted. The study was approved by the local ethical committee and informed consent was obtained from each adult participating in the study and from the parents or guardians of pediatric patients.

Patients visited the hospitals for treatments and clinical evaluations. Serum samples were collected before treatment and at different time points (week 5, 10, 25, 52, 104 and 156) during treatment. Not all patients provided blood at every time point, resulting in 872 sera in total for the SIT group.

Thirty-one patients who received only medications were included in the non-SIT control group. Serum samples were collected at weeks 0 and 52, giving 62 sera in total for the control group. Sera were stored at $-18^{\circ} \mathrm{C}$ until needed for measurements.

\section{SIT Protocol}

The patients were treated with subcutaneous injections of standardized aluminium-formulated Der p Alutard ${ }^{\circledR}$ SQ vaccine (ALK-Abello A/S, Horsholm, Denmark). The treatment protocol followed the recommended updosing schedule of 16 weeks before reaching a maintenance dose of 100,000 Alutard ${ }^{\circledR}$ SQ with a duration of 3-5 years for the SIT (fig. 1).

\section{Clinical Evaluations}

The 225 SIT patients and 31 control patients from Guangzhou were requested to complete a symptom and medication diary during the whole course of treatment. Patients were asked to rate the symptoms of asthma (daytime: $0-5$; night time: $0-4$ ) and rhinitis (day or night time: $0-2$ ) according to the severity and frequency of the symptoms in disturbing daily activities and sleep [2]. The medication score was calculated by recording a dose of $1-500 \mu \mathrm{g}$ of budesonide or the equivalent dose of inhaled corticosteroid, or $130 \mu \mathrm{g}$ of budesonide or the equivalent dose of nasal corticosteroid, each puff of salbutamol/terbutaline or the equivalent dose of another inhaled $\beta 2$-agonist and $10 \mathrm{mg}$ of oral loratadine or the equivalent dose of another antihistamine tablet. $\mathrm{FEV}_{1}$ was measured at each patient visit.

\section{In vitro Serum Der p-Specific IgE Measurements}

Der p-specific IgE in serum was measured by ImmuoCAP 100 fluorescence enzyme immunoassay system (Phadia, Uppsala, Sweden). The results are reported as $\mathrm{kU} / \mathrm{l}$, with a cut-off value of $0.35 \mathrm{kU} / \mathrm{l}$ and upper IgE detection limit of $100 \mathrm{kU} / \mathrm{l}$. Any sample with no detectable IgE level was regarded as $0.18 \mathrm{kU} / \mathrm{l}$ for data analysis. 
Fig. 1. Flow chart of the dosing schedule of injection and visits for clinical evaluation and blood sampling.

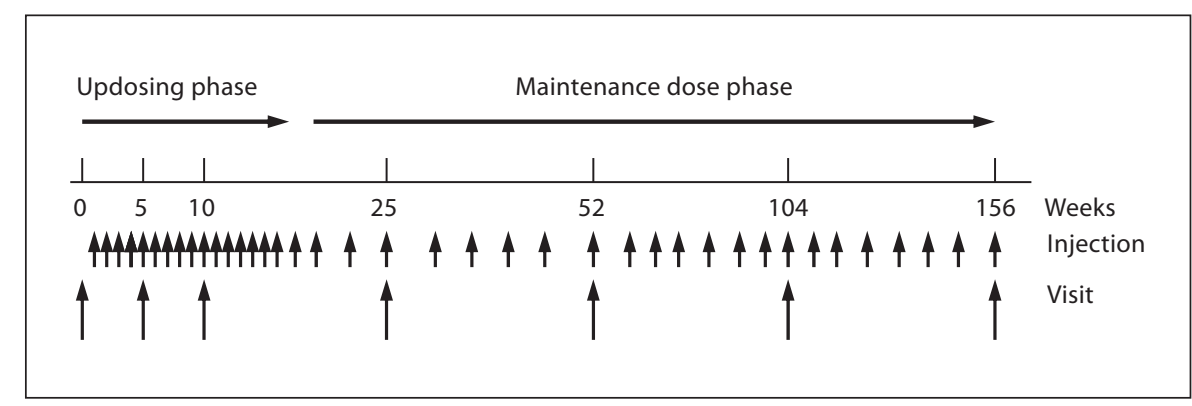

In vitro Serum Der p-Specific IgG4 Measurements

Der p-specific IgG4 in serum samples was measured by a fourlayer sandwich ELISA [13]. In brief, the plate was coated with Der $\mathrm{p}$ whole culture extract (ALK-Abello A/S) overnight at $4-8{ }^{\circ} \mathrm{C}$. The next day, at room temperature, it was incubated with diluted patient serum for $2 \mathrm{~h}$, then incubated with mouse anti-IgG4 monoclonal antibody (ALK-Abello A/S) for $1 \mathrm{~h}$. Peroxidase-labeled anti-mouse IgG (KPL Inc., Gaithersburg, Md., USA) was used for detection, and TMB substrate was used for coloring and stopped by $0.5 \mathrm{M} \mathrm{H}_{2} \mathrm{SO}_{4}$. The IgG4 concentration was read at 450 nm (EL340 Bio Kinetics reader, Bio-tek instruments Inc., Winooski, Vt., USA). The IgG4 concentration was measured as arbitrary units (AU/ml). The detection limit was $40 \mathrm{AU} / \mathrm{ml}$. For data analysis, samples with undetectable IgG4 levels were regarded as $20 \mathrm{AU} / \mathrm{ml}$, corresponding to half of the detection limit.

\section{Statistical Analysis}

GraphPad Prism 5.0 for Windows (GraphPad Software Inc., San Diego, Calif., USA) was used for the statistical analysis in this study. Mann-Whitney t test and Spearman correlation test were used with $\mathrm{p}<0.05$ defined as significant. The median value of each group was used due to no data in groups passing the normality tests.

\section{Results}

\section{Subgroups}

Table la shows the demographic and baseline data of the SIT patients $(\mathrm{n}=335)$ and medication control patients $(\mathrm{n}=31)$ in the study. The distribution of the included patients are grouped into adults and children, male and female, and subdivided into 3 disease groups: allergic rhinitis (Rh), allergic asthma (As) and allergic asthma with rhinitis (As+Rh). The number of males and females were similar for adults, while for the children $75 \%$ were male and only $25 \%$ female. There were very few patients with As alone since about $80 \%$ of As patients also had rhinitis. Among the adults there were similar numbers with $\mathrm{Rh}$ alone compared to patients with As+Rh. For children, $50 \%$ of patients were boys with As+Rh. There was no difference between the SIT and control group in specific IgE and IgG4 levels. Table $1 \mathrm{~b}$ shows the clinical data at baseline of the SIT patients from Guangzhou $(\mathrm{n}=225) \mathrm{com}$ pared with the control patient group. No differences were shown between the two groups.

\section{Symptom-Medication Scores and FEV $V_{1}$}

The mean daily symptom and medication scores of rhinitis and asthma, and change of $\mathrm{FEV}_{1} \%$ predicted at the visit time points are shown in figure $2 \mathrm{a}-\mathrm{c}$. Symptom and medication scores of rhinitis significantly declined between 0 and 10 weeks in children (3.79 \pm 0.42 to 1.51 $\pm 0.52, \mathrm{p}=0.031)$ and between 0 and 25 weeks in adults (3.65 \pm 0.65 to $1.67 \pm 0.39, \mathrm{p}=0.048)$ when compared with the respective control groups. Although the scores remained at significantly lower levels in both children and adults until week 156, children showed significantly greater improvement than adults from weeks 52-156 (p < 0.05 , fig. 2a). Symptom and medication scores of asthma in children and adults also reduced significantly after 25 weeks of SIT $(p<0.05)$ while there were no significant differences between children and adults during the whole course ( $p>0.05$, fig. $2 b$ ). However, $\mathrm{FEV}_{1} \%$ predicted in children improved significantly at weeks 104 and 156 when compared with the controls and adults $(\mathrm{p}<0.05$, fig. 2c).

The analysis of clinical changes between groups of $\mathrm{Rh}$, As and $\mathrm{Rh}+$ As could not be performed since the rating criteria was not comparable between rhinitis and asthma. The changes of the clinical data between males and females did not show a significant difference (data not shown).

\section{Concentrations of Der p-Specific IgE before SIT}

Figure 3a shows the distribution of Der p-specific IgE before immunotherapy. It shows that children have significantly higher specific IgE levels than adults ( $\mathrm{p}<$ 0.0001 , fig. 3a). There was no difference in specific $\operatorname{IgE}$ between males and females $(\mathrm{p}=0.8593)$. The specific IgE 
Table 1. Data of SIT and control patients in the study

a Demographic and baseline data of the total SIT and control patients

\begin{tabular}{lccc}
\hline & SIT group $(\mathrm{n}=335)$ & Control group $(\mathrm{n}=31)$ & $\mathrm{p}$ \\
\hline Children (5-14 years of age), n (\%) & $226(67.6)$ & $18(58.1)$ & 0.126 \\
$\quad$ Age, median (range) & $8(6-11)$ & $9(7-12)$ & 0.217 \\
$\quad$ Male/Female & $171 / 55$ & $13 / 5$ & 0.551 \\
Adults (15-64 years of age), n (\%) & $109(32.5)$ & $13(41.9)$ & 0.092 \\
$\quad$ Age, median (range) & $27(20-35)$ & $29(23-37)$ & 0.630 \\
$\quad$ Male/Female & $57 / 52$ & $7 / 6$ & 0.234 \\
Number of subjects with Rh, n (\%) & & & 0.489 \\
$\quad$ Children & $53(23.4)$ & $5(27.8)$ & 0.209 \\
$\quad$ Adults & $40(36.7)$ & $4(30.8)$ & 0.349 \\
Number of subjects with As, n (\%) & $28(12.4)$ & $3(16.7)$ & 0.761 \\
$\quad$ Children & $14(13.0)$ & $2(15.4)$ & 0.087 \\
$\quad$ Adults & $145(64.2)$ & $10(55.5)$ & 0.696 \\
Number of subjects with As+Rh, n (\%) & $55(50.5)$ & $7(53.8)$ & 0.056 \\
$\quad$ Children & $48.8(16.6-101)$ & $52.3(29.5-101)$ & 0.062 \\
$\quad$ Adults & $185(85-411)$ & $312(129-580)$ & \\
Specific IgE to Der p, median (range), kU/l & & \\
Specific IgG4 to Der p, median (range), AU/l & &
\end{tabular}

b Clinical data at baseline of the SIT patients from Guangzhou and the control group

\begin{tabular}{lccc}
\hline & SIT group $(\mathrm{n}=225)$ & Control group $(\mathrm{n}=31)$ & $\mathrm{p}$ \\
\hline Total daily asthma symptom score & $1.62 \pm 0.32$ & $1.51 \pm 0.28$ & 0.773 \\
Daily asthma medication score & $2.09 \pm 0.81$ & $2.13 \pm 0.51$ & 0.816 \\
Total daily rhinitis symptom score & $1.79 \pm 0.24$ & $1.68 \pm 0.43$ & 0.537 \\
Daily rhinitis medication score & $1.67 \pm 0.51$ & $1.70 \pm 0.45$ & 0.671 \\
FEV $\%$ predicted & $85.87 \pm 1.68$ & $86.91 \pm 1.74$ & 0.647 \\
Blood eosinophil count, $10^{6} / 1$ & $389.9 \pm 29.5$ & $391.2 \pm 32.8$ & 0.893 \\
\hline
\end{tabular}

Data are presented as mean $\pm \mathrm{SD}$.

in children shows no significant differences among the three disease subgroups, while adults with $\mathrm{Rh}$ had significantly lower Der p-specific IgE than As+Rh adults $(\mathrm{p}=0.0043$, fig. $3 \mathrm{a})$. It also shows that the difference between children and adults reached significant levels in the $\mathrm{Rh}$ group $(\mathrm{p}<0.0001)$ and the As+Rh group $(\mathrm{p}=$ 0.0066), but not in the As group.

\section{Concentrations of Der p-Specific IgG4 before SIT}

Figure $3 \mathrm{~b}$ shows the distribution of Der p-specific IgG4 before immunotherapy. It shows that children have significantly higher specific IgG4 levels than adults ( $\mathrm{p}=$ 0.0004 , fig. $3 \mathrm{~b}$ ). There is no difference in specific IgG4 between males and females $(\mathrm{p}=0.6262)$. The specific IgG4 in children shows no significant differences among the three disease subgroups, while adults with $\mathrm{Rh}$ had significantly lower Der p-specific IgG4 than As+Rh adults ( $\mathrm{p}=0.0331$, fig. $3 \mathrm{~b}$ ). It also shows that the difference between children and adults reached a significant level in the Rh group ( $\mathrm{p}=0.0067)$, but not in the As and As+Rh groups. Spearman correlation tests show a significant positive correlation between Der p-specific IgG4 and Der $\mathrm{p}$-specific IgE $(\mathrm{r}=0.511, \mathrm{p}<0.0001)$.

\section{The Der p-Specific IgG4 in the Control Group during}

1 Year of Non-SIT Treatment

The Der p-specific IgG4 level did not change in the control group when comparing weeks 0 and 52, meaning the ratio (week 52/week 0) is not significantly different from 1 (table 2). In the SIT group, the IgG4 level and ratio at week 52 is significantly higher than the control groups $(\mathrm{p}<0.0001)$. 

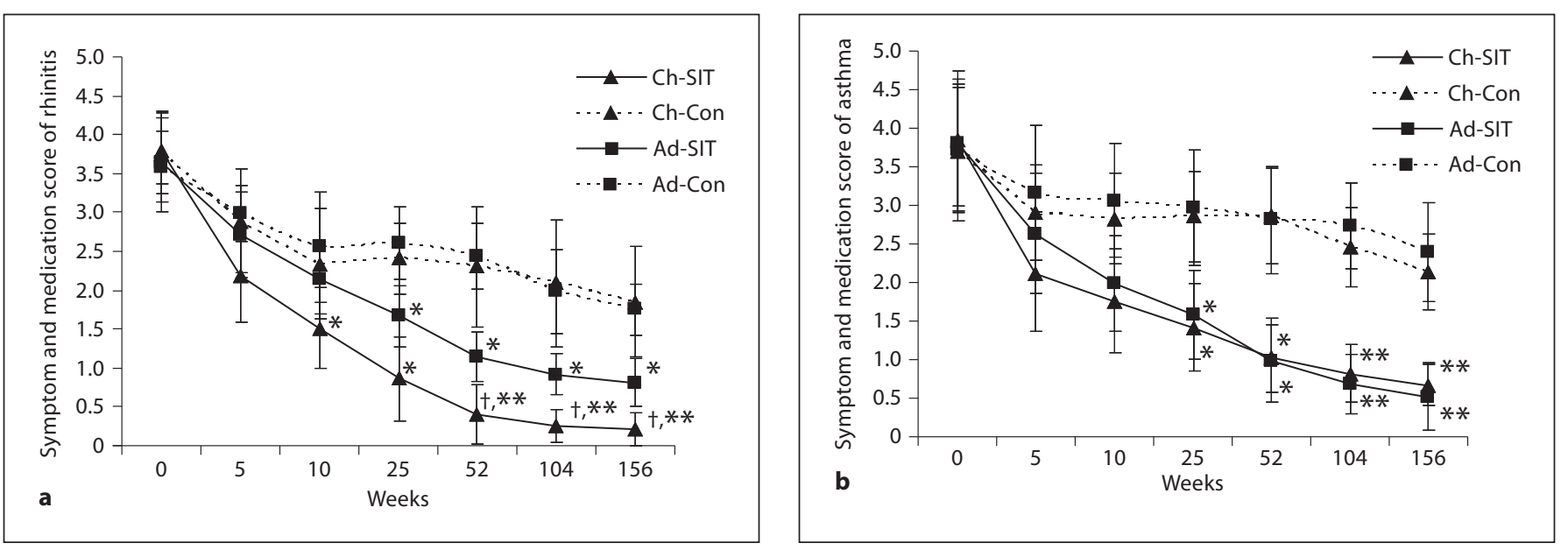

Fig. 2. Mean symptom and medication scores of rhinitis (a), asthma (b) and $\mathrm{FEV}_{1} \%$ predicted (c) in child and adult patients at visit time points. Ch-SIT $=$ Children with SIT; $\mathrm{Ch}$-Con $=$ child controls; Ad-SIT $=$ adults with SIT; Ad-Con = adult controls . ${ }^{*} \mathrm{p}<0.05,{ }^{* *} \mathrm{p}<0.01$ when compared with controls; ${ }^{\dagger} \mathrm{p}<0.05$ when compared with adults.
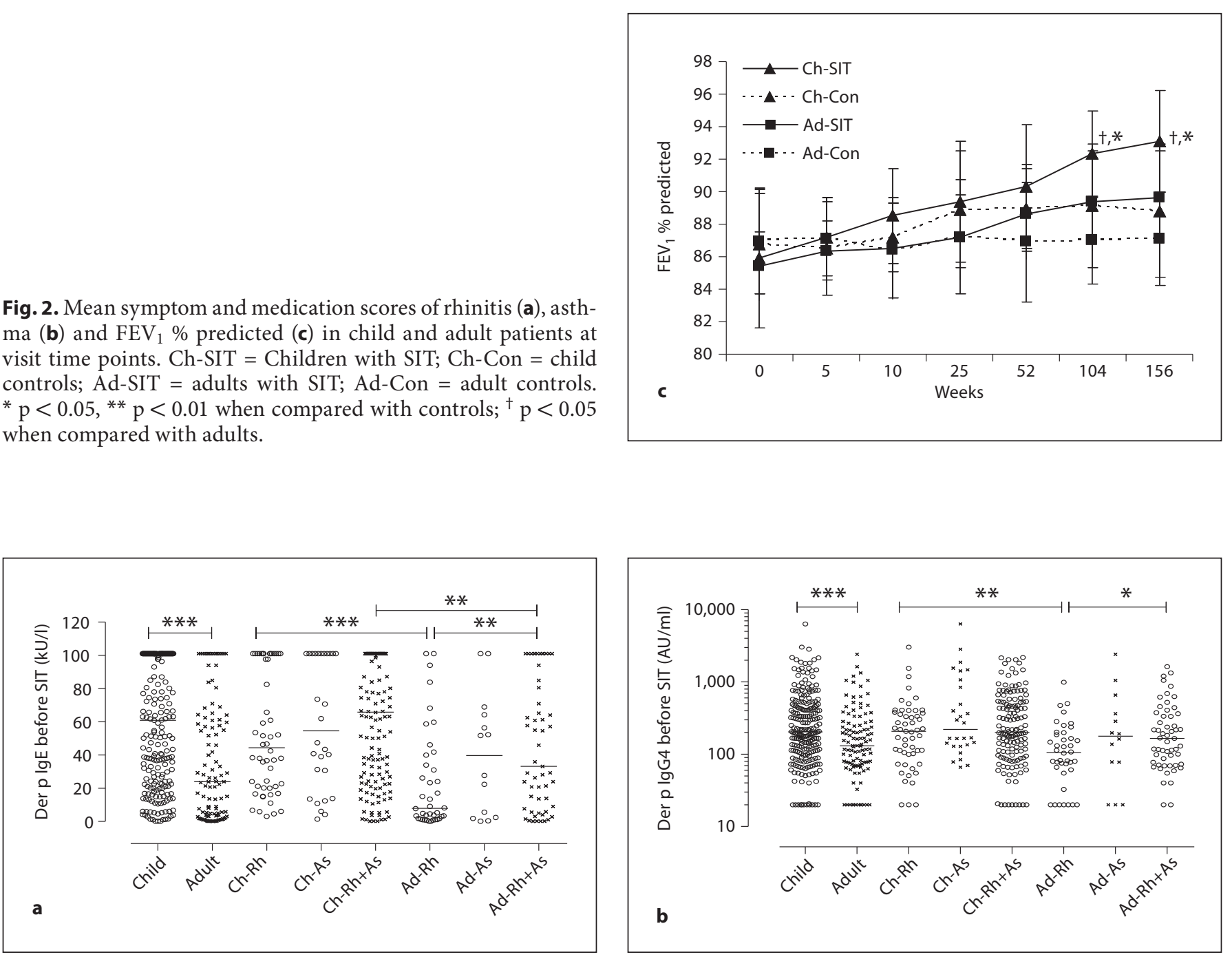

Fig. 3. The Der p-specific IgE (a) and specific IgG4 (b) levels before SIT. The horizontal line in each group indicates the median value. $\mathrm{Ch}=$ Child group; Ad = adult group. Significant differences in Mann-Whitney U test when compared between groups is shown as ${ }^{*} \mathrm{p}<0.05 ;{ }^{* *} \mathrm{p}<0.01 ;{ }^{* * *} \mathrm{p}<0.001$. The $\mathrm{y}$-axis is a log scale. 

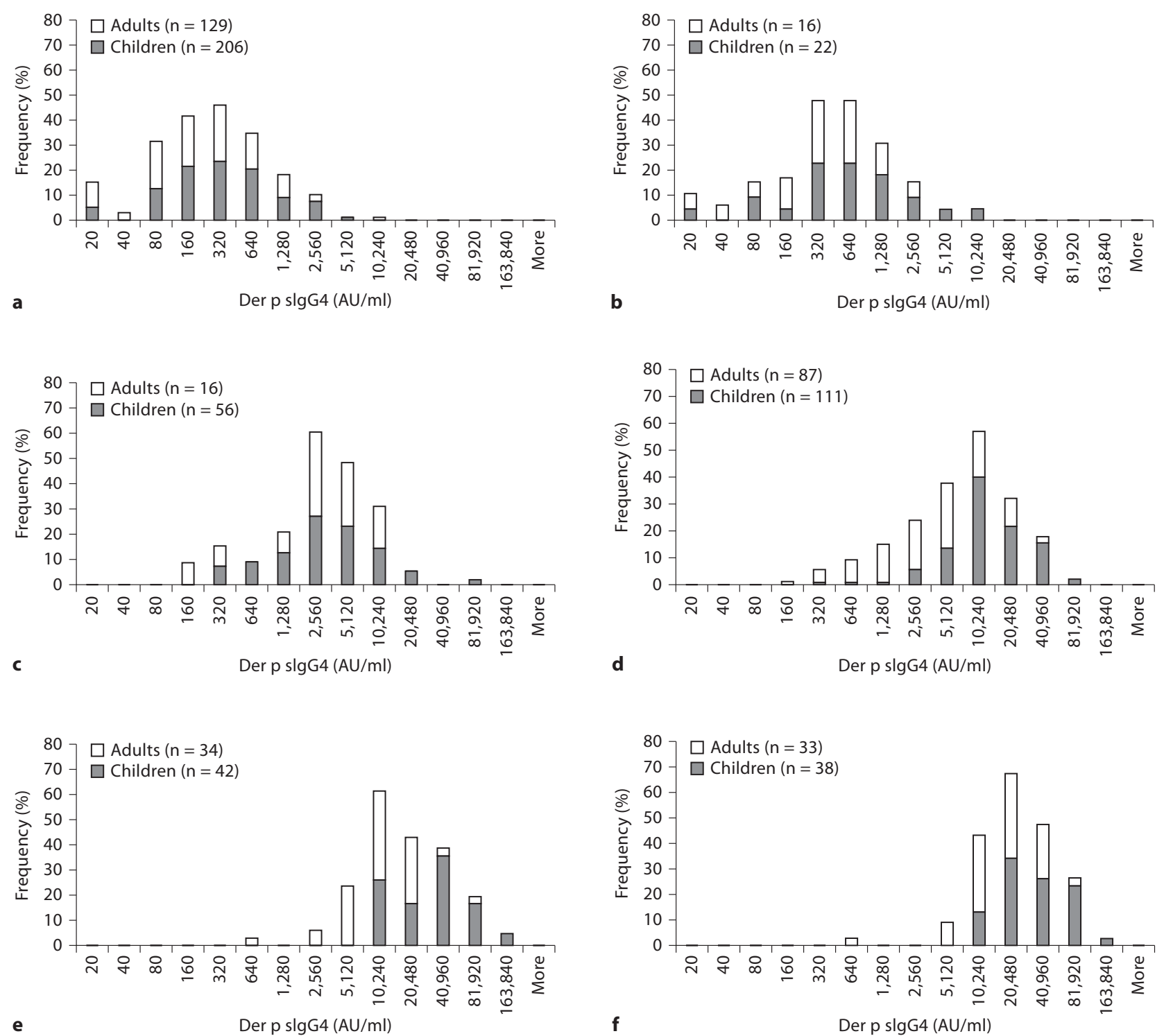

Fig. 4. The distribution of Der p-specific IgG4 levels in children and adults at different treatment times. The frequency is calculated based on the individual adult or child numbers in each group. $\mathbf{a}$ Day $0, \mathrm{n}=335$. $\mathbf{b}$ SIT 10 weeks, $\mathrm{n}=38$. c SIT 25 weeks, $\mathrm{n}=68$. d SIT 52 weeks, $\mathrm{n}=198$. e SIT 104 weeks, $\mathrm{n}=76$. f SIT 156 weeks, $\mathrm{n}=71$.

Concentration of Der p-Specific IgG4 during SIT

Figure 4 shows the distribution of Der p-specific IgG4 in children and adults at different SIT time. Before SIT, 93\% of the sera contained measurable Der p-specific IgG4 with a relatively broad distribution range of 402,560 AU/ml (fig. 4a). After starting SIT, the Der p-specific IgG4 levels increased. The distribution of specific IgG4 levels at 10 weeks (fig. $4 \mathrm{~b}$ ) had a similar range as before SIT, but there were more sera in the higher levels of
5,120-10,240 AU/ml. After 10 weeks of SIT, the IgG4 levels were significantly higher than week 0 ( $t$ test, $\mathrm{p}=0.012$ ), thus the increase of Der p-specific IgG4 was detectable from this time point. This increase is more and more obvious after 25, 52 and 104 weeks of SIT (fig. 4c-e). At SIT 156 weeks (fig. 4f), the range of Der p-specific IgG4 levels was narrower and the majority were between 10,240 and $81,920 \mathrm{AU} / \mathrm{ml}$, corresponding to a 40-250-fold increase compared to the level before starting SIT. 

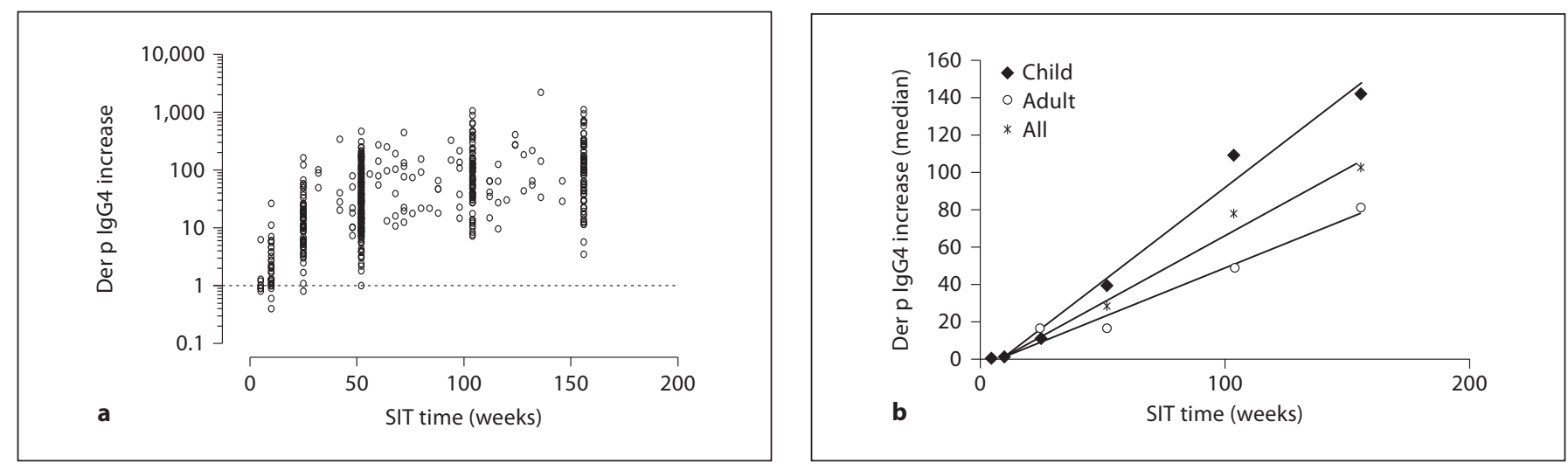

Fig. 5. The increase of Der p-specific IgG4 during SIT. The increase of IgG4 = IgG4 after SIT/IgG4 before SIT. a The increase of Der p-specific IgG4 during SIT $(n=537)$; the $y$-axis is a log scale. $\mathbf{b}$ The correlation between the increase of Der p-specific IgG4 (median) and SIT time points (weeks).

Table 2. The Der p-specific IgG4 level at week 0 (before treatment) and week 52 (after 1 year of treatment) in the control and SIT groups

\begin{tabular}{llcl}
\hline \multirow{2}{*}{ Group } & \multicolumn{2}{l}{ Specific IgG4 level, AU/ml } & Specific IgG4 ratio (week 52/week 0) \\
\cline { 2 - 3 } & week 0 & week 52 & \\
\hline Control $(\mathrm{n}=31)$ & $312(450)$ & $280(312)$ & $0.9(0.4)$ \\
SIT $(\mathrm{n}=198)$ & $165(288)$ & $6,589(8,916)^{*}$ & $28.2(55.0)^{*}$ \\
\hline
\end{tabular}

Data are median values with interquartile range in parentheses. Control group: patients only received medication treatment but no SIT for 52 weeks; SIT group: patients received Der p SIT for 52 weeks. ${ }^{*} \mathrm{p}<0.0001$. IgG4 levels are significantly different in the SIT group at week 52 versus week 0 . The IgG4 ratio is significantly different in the SIT group versus the control group.

The Der p-specific IgG4 distribution in the child and adult groups shows that the levels of specific IgG4 in children were higher compared to adults during the whole duration of treatment (fig. $4 \mathrm{a}-\mathrm{e}$ ). The trend becomes more obvious at SIT 52 and 104 weeks.

\section{Increase of Der p-Specific IgG4 during SIT}

The increase of IgG4 during SIT is defined as the ratio between the IgG4 levels after SIT at different treatment times and before SIT in sera derived from the same patient. In figure 5a, the increase of Der p-specific IgG4 in different sera, shown as the median value, is plotted against treatment time. The increase did not reach statistical significance at 5 weeks of treatment. After 10 weeks of SIT $(\mathrm{n}=38)$ the median IgG4 increase was 1.7 ( $\mathrm{p}<$ 0.001 ), representing the earliest detectable increase with statistical significance. The Der p-specific IgG4 increase reached 11.3 at SIT 25 weeks $(n=68)$, and 102 at SIT 156 weeks $(n=71)$.

When plotting the median values of Der p-specific IgG4 increase against its corresponding SIT time point, a linear correlation was obtained (fig. 5b). Further division into child and adult subgroups shows that the Der p-specific IgG4 increase in children was higher than in adults.

Figure 6 shows the pattern of the Der p-specific IgG4 increase in different disease subgroups after 52, 104 and 156 weeks of SIT. A significant difference of IgG4 increase between SIT week 52 and week 104 was found in Rh children $(\mathrm{p}=0.0041)$ and adults $(\mathrm{p}=0.0036)$, and As+Rh children $(\mathrm{p}=0.0012)$ and adults $(\mathrm{p}=0.0400)$. No significant difference was found between 104 and 156 weeks of treatment in all the subgroups. Any significant 
Fig. 6. The Der p-specific IgG4 increase in different disease subgroups (a-c) during 1, 2 and 3 years of SIT. Significant difference in Mann-Whitney $U$ test when compared between groups is shown as ${ }^{*} \mathrm{p}<0.05 ;{ }^{* *} \mathrm{p}<0.01$. $\mathrm{Ch}=$ Child group; $\mathrm{Ad}=$ adult group.

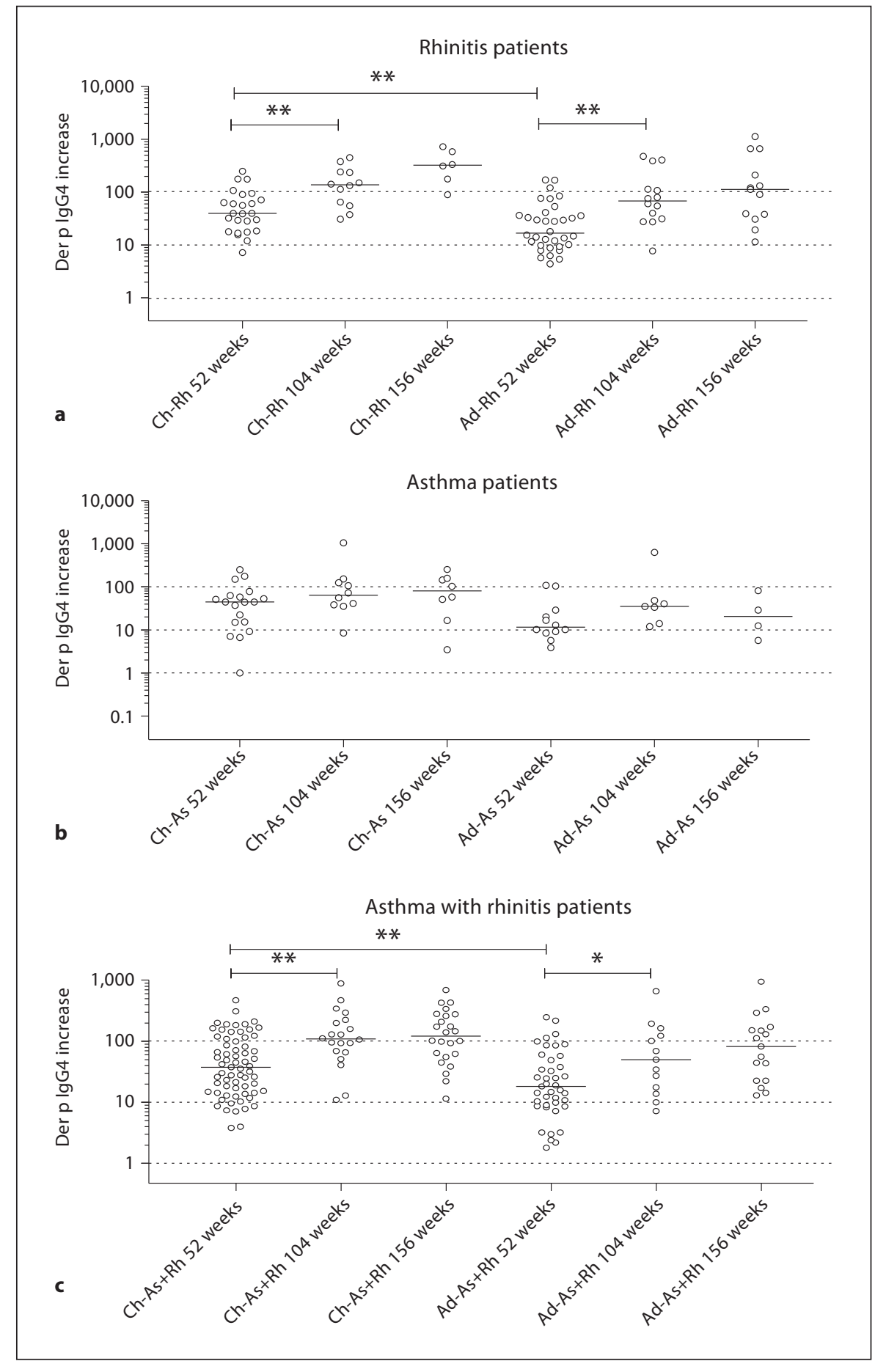

differences between age subgroups were found at SIT week 52 between Rh children and adults $(\mathrm{p}=0.0082)$ and As+Rh children and adults $(\mathrm{p}=0.0077)$. The significant differences in disease subgroups were found at SIT week 156 , between $\mathrm{Rh}$ and As children $(\mathrm{p}=0.0127), \mathrm{Rh}$ and
As+ $+\mathrm{Rh}$ children $(\mathrm{p}=0.0459)$, and $\mathrm{Rh}$ and As adults $(\mathrm{p}=$ 0.0474 ). The Der p-specific IgG4 increase pattern in gender subgroups shows no significant difference between males and females at all the SIT time points investigated (data not shown). 


\section{Discussion}

The clinical data of the current study show that house dust mite SIT in patients with allergic rhinitis and asthma results in a significant improvement in symptoms and reduction in medication requirements in both children and adults, while children showed significantly greater improvement with rhinitis and pulmonary function than adults. In this study population, $93 \%$ of patients had measurable serum Der p-specific IgG4 before SIT, most likely induced by natural exposure to house dust mite. Spearman analysis showed strong correlation between initial specific IgG4 and sIgE against Der p. Rossi and Monasterolo [15] suggested that subjects without sIgE against a particular allergen fail to mount a significant IgG4 response; however, this was not supported by the findings of Jutel et al. [16]. Before SIT, the amount and/or affinity of specific IgG4 is probably too low to compete with $\operatorname{sgE}$ for allergen binding. The initial Der p-specific IgG4 remained unchanged when patients received only medication treatment for a duration of 1 year, confirming that medication treatment cannot induce or enhance levels of specific IgG4. During SIT, we saw a substantial increase in specific IgG4, while sIgE remained unchanged [13]. This indicates that during high-dose SIT using standardized vaccines, the immune system is induced to produce high levels of specific IgG4. Along with the improvement of clinical symptoms and reduction of medication during the course in SIT patients, specific IgG4 can therefore be used as a biomarker for a specific immune response against the SIT vaccine being administrated.

The magnitude of increase in Der p-specific IgG4 concentrations is dependent on the concentration of the allergenic extract used for immunotherapy [17] and on the duration of treatment [13], which can be linked to the accumulated dose being administrated. It is well established that low dose subcutaneous SIT is clinically ineffective [3]. We detected a significant increase of IgG4 10 weeks after SIT, at the time point when patients reached the end of the updosing period and both symptoms and medications started to be reduced. The production of IgG4 is stimulated by cytokine IL-10 produced by regulatory T cells. Francis et al. [18] showed that production of IL-10 precedes clinical symptom relief and IgG4 production in patients undergoing subcutaneous grass pollen immunotherapy, detecting a significant increase of IL-10 after 2-8 weeks of SIT.

Among the investigated patient population, the level of Der p-specific IgG4 reached statistical significance after 10 weeks of subcutaneous Der p SIT and the level contin- ued to increase during the recommended treatment period. The majority of patients reached about a 100 -fold increase of Der p-specific IgG4 serum concentrations after 3 years of treatment when symptom and medication scores continued to reduce and remained at low levels. Through a large sample size, this investigation shows a clear linear and predictable correlation between the increase of Der p-specific IgG4 levels and duration of Alutard ${ }^{\circledR}$ SIT, and a decrease in the frequency and severity of symptoms and usage of medications. The increased ratio of specific IgG4 is a better indicator than the absolute level of specific IgG4, since it removes the influence of initial specific IgG4 levels before SIT and only reflects the magnitude of specific IgG4 increase. Through the correlation shown in figure $5 \mathrm{~b}$, it is possible to roughly estimate the expected increase of specific IgG4 during SIT. This could be part of the evaluation of the treatment performance as one objective parameter reflecting a specific immune response towards the allergenic extracts being administrated during SIT.

This investigation shows that children have higher initial sIgE and specific IgG4 than adults before SIT, which agrees with the findings of Hales et al. [19]. Children had a significantly higher increase of Der p-specific IgG4 than adults after 1 year of SIT, and this finding matches our clinical evaluations in which children demonstrated greater improvement as compared to adults and their respective controls, showing that children are more effective than adults in producing specific IgG4 at the early stage of SIT. The relatively higher increase of IgG4 in children compared to adults could be influenced by a higher ratio of vaccine dose to body weight in children. However, it also indicates that performing SIT in early age might be more beneficial, because the immune system may respond better to the treatment when it is still under development.

With respect to different diseases, before SIT, adult rhinitis patients had significantly lower Der p-specific IgE and IgG4 levels compared to patients with both asthma and rhinitis, while the levels in children showed no significant correlation to the disease severity. However, during 36 months of SIT, rhinitis patients, both adult and child, showed a significantly higher increase of specific IgG4 compared to asthma patients and patients with both asthma and rhinitis. The reason for this change is not completely understood, but we assume that the immune system in patients with milder allergic symptoms is more effective in producing IgG4. Niggeman et al. [20] showed that SIT can prevent the development of asthma in children with rhinitis as well as prevent the development of new allergen sensitivities. Our data suggest the beneficial effect of SIT in the early stage of disease. 


\section{Conclusion}

The level of specific IgG4 induced by SIT may not directly correlate to the clinical efficacy of SIT; however, it reflects a specific response of the immune system and its ability to produce this blocking antibody. The first detectable changes in the increase of specific IgG4 during subcutaneous SIT occur after 10 weeks of treatment. Untreated allergic children have higher levels of $\operatorname{IgE}$ and IgG4 compared to adults and during the first year of SIT the IgG4 levels increases faster in children than in adults. Rhinitis patients have a higher increase in levels of IgG4 than patients with more severe allergic symptoms. Our data suggest the beneficial effect of SIT in the early stages of disease.

\section{Acknowledgements}

The authors thank Mulin Feng, Nili Wei, Tiantian Liu and Haifeng Zhong at ALK-GIRD lab, Guangzhou, China, for excellent technical assistance, and Patrice Biossy at ALK Spain for providing anti-IgG4 reagent. The study is partly supported by Chinese National Science Funding (30972808).

\section{References}

1 Bousquet J, Lockey R, Malling HJ: Allergen immunotherapy: therapeutic vaccines for allergic diseases: a WHO position paper. J Allergy Clin Immunol 1998;102:558-562.

$\checkmark 2$ Wang H, Lin X, Hao C, Zhang C, Sun B, Zheng J, Chen P, Sheng J, Wu A, Zhong N: A double-blind, placebo-controlled study of house dust mite immunotherapy in Chinese asthmatic patients. Allergy 2006;61:191-197.

-3 Larché M, Akdis CA, Valenta R: Immunological mechanisms of allergen-specific immunotherapy. Nat Rev Immunol 2006;6: 761-771.

-4 Jutel M, Akdis CA: Immunological mechanisms of allergen-specific immunotherapy. Allergy 2011;66:725-732.

5 Akkoc T, Akdis M, Akdis CA: Update in the mechanisms of allergen-specific immunotherapy. Allergy Asthma Immunol Res 2011; 3:11-20.

-6 Aalberse RC, Stapel SO, Schuurman J, Rispers T: Immunoglobulin G4: an odd antibody. Clin Exp Allergy 2009;39:469-477.

7 Van Neerven RJ, Knol EF, Ejrnaes A, Würtzen PA: IgE-mediated allergen presentation and blocking antibodies: regulation of $\mathrm{T}$ cell activation in allergy. Int Arch Allergy Immunol 2006;141:119-129.

-8 Royer B, Varadaradjalou S, Saas P, Guillosson JJ, Kantelip JP, Arock M: Inhibition of IgE-induced activation of human mast cell by IL-10. Clin Exp Allergy 2001;31:694-704.
9 James LK, Shamji MH, Walker SM, Wilson DR, Wachholz PA, Francis JN, Jacobson MR, Kimber I, Till SJ, Durham SR: Long-term tolerance after allergen immunotherapy is accompanied by selective persistence of blocking antibodies. J Allergy Clin Immunol 2011; 127:509-516.

10 Li J, Sun B, Huang Y, Lin X, Zhao D, Tan G, Wu J, Zhao H, Cao L, Zhong N: China Alliance of Research on Respiratory Allergic Disease: a multicentre study assessing the prevalence of sensitizations in patients with asthma and/or rhinitis in China. Allergy 2009;64:1083-1092.

11 Zhang C, Gjesing B, Spangfort MD, Xu J, Zhong N: The allergen-specific IgE reactivity pattern of Chinese house dust mite allergic patients. Allergy 2008;63:1640-1641.

-12 Zheng YW, Li J, Lai XX, Zhao DY, Liu XF, Lin XP, Gjesing B, Palazzo P, Mari A, Zhong NS, Spangfort MD: Allergen micro-array detection of specific IgE reactivity in Chinese allergic patients. Chin Med J 2011;124:43504354.

13 Xiao X, Wang D, Cui B, Lai X, Gjesing B, Spangfort M, Zhong N: The effect on Der $\mathrm{f}$ specific IgG4 of mite allergic patient after Der p specific immunotherapy (in Chinese). Chin J Allergy Clin Immunol 2009;3:34-38.

14 Thomas WR, Smith WA, Hales BJ, Mills KL, O'Brien RM: Characterization and immunobiology of house dust mite allergens. Int Arch Allergy Immunol 2002;129:1-18.

15 Rossi RE, Monasterolo G: Evaluation of recombinant and native timothy pollen ( $\mathrm{rPhl} \mathrm{p}$ $1,2,3,7,11,12$ and $n P h l p ~ 4)$-specific IgG4 antibodies induced by subcutaneous immunotherapy with timothy pollen extract in allergic patients. Int Arch Allergy Immunol 2004; 135:44-53.
6 Jutel M, Jaeger L, Suck R, Meyer H, Fiebig H, Cromwell O: Allergen-specific immunotherapy with recombinant grass pollen allergens. J Allergy Clin Immunol 2005;116:608613.

17 Van Metre TE Jr, Adkinson NF Jr, Lichtenstein LM, Mardiney MR Jr, Norman PS Jr, Rosenberg GL, Sobotka AK, Valentine MD: A controlled study of the effectiveness of the Rinkel method of immunotherapy for ragweed pollen hay fever. J Allergy Clin Immunol 1980;65:288-297.

18 Francis JN, James LK, Paraskevopoulos G, Wong C, Calderon MA, Durham SR, Till SJ: Grass pollen immunotherapy: IL-10 induction and suppression of late responses precedes IgG4 inhibitory antibody activity. J Allergy Clin Immunol 2008;121:1120-1125.

19 Hales BJ, Martin AC, Pearce LJ, Laing IA, Hayden CM, Goldblatt J, Le Souëf PN, Thomas WR: IgE and IgG anti-house dust mite specificities in allergic disease. J Allergy Clin Immunol 2006;118:361-367.

20 Niggemann B, Jacobsen L, Dreborg S, Ferdousi HA, Halken S, Høst A, Koivikko A, Koller D, Norberg LA, Urbanek R, Valovirta E, Wahn U, Möller C; PAT Investigator Group: Five-year follow-up on the PAT study: specific immunotherapy and longterm prevention of asthma in children. Allergy 2006;61:855-859. 